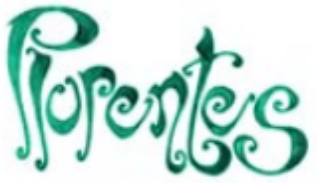

Artes y Letras
Plurentes. Artes y Letras

ISSN: 1853-6212

plurentesunlp@gmail.com

Universidad Nacional de La Plata

Secretaría de Asuntos Académicos

Prosecretaría de Asuntos Académicos

Bachillerato de Bellas Artes, "Prof. Francisco A. De Santo"

Argentina

\title{
Gestionar sonidos, compartir sentidos: aprender a aprender desde la clase de instrumento
}

\author{
Dicundo, Carlos \\ Gestionar sonidos, compartir sentidos: aprender a aprender desde la clase de instrumento \\ Plurentes. Artes y Letras, núm. 11, e010, 2020 \\ Universidad Nacional de La Plata \\ Secretaría de Asuntos Académicos \\ Prosecretaría de Asuntos Académicos \\ Bachillerato de Bellas Artes, "Prof. Francisco A. De Santo" \\ Argentina \\ DOI: https://doi.org/10.24215/18536212e010
}

Atribución no comercial compartir igual (CC BY-NC-SA) 4.0 


\title{
Gestionar sonidos, compartir sentidos: aprender a aprender desde la clase de instrumento
}

Manage sounds, share senses: learning to learn in instrument classes.

Carlos Dicundo

DOI: https://doi.org/10.24215/18536212e010

Bachillerato de Bellas Artes, Universidad Nacional de La

Plata, Argentina

cmdicundo@hotmail.com

Recepción: 14 Septiembre 2020

Aprobación: 18 Septiembre 2020

Publicación: 26 Octubre 2020

\section{RESUMEN:}

El siguiente artículo conserva la estructura y contenido de una propuesta pedagógica producida en el marco de los Concursos Docentes de los colegios de pregrado de la UNLP. La temática gira en torno a la enseñanza del instrumento piano dentro del Bachillerato de Bellas Artes "Prof. Francisco A. De Santo" de la ciudad de La Plata. Desde una concepción socio-constructivista de la enseñanza y el aprendizaje instala la competencia Aprender a Aprender como eje central para el desarrollo de la subjetividad y autonomía del estudiante.

Palabras Clave: Aprendizaje, Enseñanza, Piano, Metacognición, Autonomía.

\begin{abstract}
:
The following article preserves the structure and content of a pedagogical proposal written for the UNLP undergraduate schools Teaching Recruitment and Selection Process. The theme revolves around the teaching of piano at the "Prof. Francisco A. De Santo" School of Fine Arts in the city of La Plata. From a socio-constructivist conception of teaching and learning, it sets the Learn to Learn competence as the central axis for the development of students' subjectivity and autonomy.
\end{abstract}

KEYWORDS: Learning, Teaching, Piano, Metacognition, Atonomy.

Resulta todo un desafío pensar una clase de instrumento que respete los principios y políticas educativas que tanto la UNLP como el Bachillerato de Bellas Artes (BBA) promueven en sus textos fundamentales. La clase de piano se carga de imágenes remotas y nostálgicas en el común de las personas: célebres maestros adustos revelando conocimiento custodiado por escuelas pianísticas; el reflejo de un alumno inmóvil, pasivo y receptivo frente a un objeto de lujo poco popular, perteneciente a los sectores más acomodados de la sociedad; la partitura en el atril exhortando fidelidad incondicional, imponiendo un único objetivo, su "correcta" ejecución; la imagen del pianista bohemio apartado e incomprendido; modelos de músicos difundidos y exigidos por la industria cultural, modelos exitistas y espectaculares de relacionarse con la música y el arte.

Superar todas estas determinaciones supone proponer alternativas más profundas y sensibles, significa ubicar a la educación secundaria obligatoria, especializada en arte, en un lugar vital, más amplio, y menos estereotipado. En este sentido la UNLP orienta sus diferentes dependencias con políticas de inclusión que tienden a facilitar el ingreso, la permanencia y el egreso sobre la base de una universidad nacional, pública, gratuita, laica y abierta. Entendiendo a la educación como derecho, los colegios de pregrado como es el Bachillerato de Bellas artes articulan acciones concretas frente a la diversidad que genera el ingreso por sorteo, mecanismo inmensamente democrático alcanzado en la historia más reciente de los colegios dependientes de la UNLP.

El proyecto académico y de gestión actual, el plan 2012, y la propuesta de gestión departamental del BBA junto con el programa vigente de la materia (instrumento piano) proponen con un sólido cuerpo teórico y con 
prácticas concretas trabajar a favor de estos principios fundamentales generando un marco de referencia muy valioso que nos sitúa a los docentes en un contexto especial y particular y nos inspira para generar prácticas educativas democráticas e innovadoras que permitan superar "formas", concepciones y modelos instalados como incuestionables.

\section{APRENDER A APRENDER}

Mi propuesta pedagógica sitúa al alumno como protagonista del proceso de aprendizaje, centrándose en el desarrollo de la competencia de aprender a aprender (AA) como una hipótesis de trabajo a desplegar en los tres años de ESB. Considerando las trayectorias individuales de cada alumno, sería erróneo proponer plazos o secuencias fijas según se trate de primero, segundo o tercer año de ESB, en cambio se considera el desarrollo de esta competencia y su consecuente aprendizaje autónomo como grados crecientes sin poner topes preestablecidos.

Considero que el AA puede constituirse en una trama de trabajo armónico y transversal con respecto a las propuestas departamentales e institucionales colaborando con el desarrollo de la competencia comunicativa, la subjetividad e intersubjetividad y acceder de una manera más eficiente y consciente al campo de la producción.

La competencia de aprender a aprender emerge de la concepción socio-constructivista de la enseñanza y el aprendizaje. Desde esta concepción se concibe el aprendizaje como un proceso de construcción de significados y de atribución de sentido mediante la modificación de esquemas de conocimiento. Implica conectar el conocimiento previo del alumno con el nuevo conocimiento para generar un aprendizaje significativo.

La competencia AA es por lo general entendida desde dos enfoques:

- enfoque practicista, más tradicional y utilitario, se centra en el aprendizaje de técnicas y procedimientos que garantizan alcanzar de modo más eficiente los objetivos propuestos.

- enfoque estratégico. Engloba y supera al enfoque anterior. Valora los procesos metacognitivos con los que el alumno toma conciencia de su situación como estudiante y lo impulsa a gestionar su propio aprendizaje.

Es frecuente encontrar alumnos que ignoran qué tienen y cómo tienen que aprender. O más aún alumnos que dudan si en el terreno del arte o la música hay algo que aprender apareciendo así la dualidad cognitivo/ intuitivo tan arraigada donde la música queda solo en el plano de lo intuitivo o el talento.

El enfoque estratégico permite que el alumno vea el proceso subyacente, reflexione y tome decisiones para gestionar de modo autónomo su propia actividad musical. Este enfoque no solo afecta a los alumnos sino que promueve otras formas de enseñar, cuya meta no solo es que los docentes enseñemos piano, a ejecutar "correctamente" una obra, a brindar herramientas técnicas, a contextualizar estilísticamente (y digo no solo), sino también, y sobre todo, enseñar a aprender, enseñar a regular metacognitivamente la propia acción de los alumnos y, volviendo a las bases del constructivismo, ayudarlos a construir conocimiento.

El gran aporte de esta concepción es transformar la reflexión en objetivo de la enseñanza y utilizar los contenidos musicales como un medio para alcanzar esa meta. Los contenidos son el medio y no el fin.

\section{SubJeTIVIDAD- INTERSUBJETIVIDAD. MotivaCióN}

La adolescencia temprana supone el inicio de un complejo proceso de estructuración y de construcción de la subjetividad. Ser, para los adolescentes se presenta como una incógnita y un desafío y fracasar en esta tamaña empresa existencial deriva en "fenómenos de desubjetivación que implican vivencias de desapropiación 
y vaciamiento emocional e intelectual, anomia o sujetamiento a pautas arcaicas o infantiles, bloqueos o directamente detención en el desarrollo" (Efrón, 1996, p. 32)

Afortunadamente la subjetivación conlleva la presencia del otro. Aparecen nuevos modelos de adultos, entre ellos los docentes, junto con referentes horizontales: los grupos de pares, los compañeros. La importancia de la intersubjetividad, de los intercambios que proporciona la grupalidad contribuye a consolidar la identidad adolescente. En este sentido la idea de aula abierta impulsada desde la institución permite habitar espacios compartidos, lugares de encuentros y sociabilización de saberes que favorecen "el fortalecimiento de los procesos de construcción de identidad y autoestima” (Ley 26.150 de ESI, 2006). La producción áulica trasciende los límites del piano, del aula y de la propia institución.

Tal como se plantea en el proyecto de gestión la elección constituye un concepto nodal en la construcción de la subjetivación puesto que los alumnos pueden decidir qué trayectos académicos quieren transitar: optar por un instrumento, elegir materias, orientaciones, etc.

Fomentar la capacidad de aprender a aprender es generar en los alumnos sentimientos de competencia para los cuales la motivación representa el principal motor. Las nuevas concepciones del aprendizaje incluyen a la motivación como "un proceso que engloba factores cognitivos y afectivos que van a determinar la elección, iniciación, dirección, magnitud y calidad de una acción que persigue alcanzar un fin determinado" (Lanz, 2006, p. 124). Es necesario superar la utilización de motivaciones externas (aprobar, obtener reconocimiento, conformar al docente) y promover una implicación del alumno más auténtica y movilizadora mediante una motivación intrínseca, que impulse una acción con autodeterminación (el control de la acción depende de uno mismo), competencia (sentirse capaz) y satisfacción de hacer algo que se quiere.

\section{COMUNiCACión EMOCIONAL}

En relación con el apartado anterior, la comunicación ocupa un lugar central ya que en el encuentro con el otro el estudiante se sigue encontrando a sí mismo. La interpretación musical vista desde acá no puede ser entendida como una creación o reproducción ajena.

$\mathrm{Al}$ comunicarnos con nuestro lenguaje cotidiano recurrimos de manera intuitiva al sonido, los gestos y movimientos para amplificar un sentido, para conducir emociones. Las emociones "no nos dicen nada concreto sobre el mundo, para eso es más eficaz el lenguaje de las palabras, pero nos hacen sentir algo sobre el mundo que nos lleva a comportarnos de una u otra forma". (Torrado-Braga, 2016, p. 112)

Las tradiciones musicales supieron entender la importancia de la expresividad musical ligada a la comunicación, pero muchas veces relegaban al talento individual como forma de conseguirla, la expresividad como mecanismo de exclusión: se es o no se es expresivo, como si existieran índices formalizados o categorías misteriosas de expresividad.

Con todo sería indispensable entender que la comunicación emocional no es "inocente" nos ayuda a relacionar conductas con sensaciones y a impactar en las sensaciones de otros. Desde esta propuesta se entiende a la música como una manera de gestionar sonidos para impactar emocionalmente en el oyente, y el desafío pedagógico reside en volver estratégico el conocimiento intuitivo de los alumnos, la música como herramienta para el aprendizaje estratégico de la comunicación emocional. La expresividad se materializa y se transforma en una variable concreta de aprendizaje que acepta una acción pedagógica y un posible desarrollo.

La producción instrumental es el medio artístico con el cual el alumno sensibiliza su propia voz. Para esto es necesario superar concepciones conservadoras acerca de la interpretación musical.

La concepción o teoría directa reduce el aprendizaje a los resultados, enseñar produce directamente aprendizaje por lo cual interpretar es seguir los saberes dados por el profesor para ejecutar correctamente una obra, decodificarla, acercarse lo más posible a una reproducción ideal siendo este un fin en sí mismo. El centro puesto en el maestro. 
La teoría interpretativa resalta la importancia de promover los dominios técnicos que hacen posible una ejecución correcta y los procesos cognitivos que ayudan a gestionar esos procedimientos técnicos (atención, comprensión, etc.). El centro puesto en el instrumento ya que dominar el instrumento hace posible reproducir la partitura correctamente.

En ambas teorías los procesos motores cognitivos del alumno se gestionan externamente.

Una concepción pensada desde el constructivismo ubica al alumno en una posición activa frente a la interpretación musical impulsándolo a reflexionar sobre su propia práctica y tomar decisiones. La interpretación no es una instancia posterior a la decodificación o dominio técnico, sino que por el contario es el punto de referencia para la selección, por parte del alumno, de los procesos cognitivos y procedimentales más convenientes a poner en juego.

Interpretar es subjetivar, recrear el texto musical cargándolo con significaciones propias del alumno/ intérprete que in-corpora, en sentido literal, conocimiento encarnado sentido, vivido asimilado como parte de su cuerpo para luego comunicarlo.

Cada obra pianística presenta un cuerpo de contenidos que funcionan de manera integrada y particular dentro del contexto global que plantea cada obra musical La producción instrumental orienta esos contenidos hacia un uso del conocimiento como práctica, es decir, se aprenden mediante la práctica, la experiencia directa y se significan en ella. De esta manera los contenidos de carácter procedimental adquieren una considerable relevancia priorizando el "saber cómo": metodologías, procedimientos, técnicas y habilidades para plantear y resolver un problema.

Según el eje de esta propuesta los contenidos serán el medio por el cual se pueda llegar a una comprensión más reflexiva del proceso de aprendizaje, es decir, no es el fin saber o dominar tal o cual contenido sino que lo que más interesa es entender metacognitivamente por qué, para qué, cómo, de qué manera, cuándo es necesario acceder a determinado contenido. En todo caso el enunciado de estas preguntas se consolida como el contenido primordial.

Se propone una secuenciación de los contenidos de forma espiralada, avanzando en el desarrollo conceptual pero volviendo siempre en sí mismos de manera diferente. Conceptos anteriores son revisados y resignificados, lo que se aprende con anterioridad, puede ser retomado y adaptado a nuevos contextos.

\section{Metodología}

La clase individual de piano es un espacio ideal para relacionarse de manera crítica y reflexiva con el conocimiento musical. Producir discursos musicales con el instrumento demanda al alumno tomar decisiones para integrar de manera orgánica los saberes propios del piano entre sí, y aquellos que provienen del resto de las materias de la formación específica (historia de la música, fundamentos musicales y composición), y realizar una síntesis que incluya y esté atravesada por su propio mundo interior.

Para desplegar la competencia AA en los tres años de ESB es importante en una primera etapa conectar con los conocimientos previos del alumno para planificar y regular la intervención del docente. Según el caso será necesaria una actividad más o menos dirigida.

Partir de situaciones de enseñanza donde se verbalizan y presentan diferentes procesos de resolución de un problema. Estas primeras etapas conforman un muestrario con las decisiones más relevantes que se pueden tomar para resolver una tarea.

Acerca de esta actividad:

Es importante notar que no se trata sólo de mostrar los pasos que es preciso seguir, como ocurre en el modelado de inspiración conductista, sino también de razonar porqué es preferible efectuar esos pasos y no otros explicitar a qué variables hay que prestar atención para proceder correctamente, cómo se escogen y por qué diferentes procedimientos de actuación, qué procedimientos alternativos podrían también emplearse, cómo se controla el proceso seguido, de qué forma y cómo uno es capaz de reconocer que ya ha resuelto la tarea, etc. (Monereo, Pozo, Castelló, 2001, p.15). 
Vemos como el concepto de elección tiene alcances metodológicos y reaparece aquí como una variable fundamental en el proceso de toma de conciencia y de gestión del propio aprendizaje. Aprender a aprender implica elegir posibilidades, caminos de resolución.

Es probable que en el principio de este proceso los alumnos asuman una posición de mayor dependencia para resolver tareas que tal vez le resulten desconocidas, que no sepan que hacer o desconozcan su capacidad de resolución de diferentes problemáticas frente a nuevas situaciones (por ejemplo nuevo repertorio, un estilo diferente del abordado hasta el momento, desarrollo de una textura, control de la sonoridad, utilización de recursos técnicos, etc.). Podrá preguntarse: ¿cuál es la forma correcta de hacer esto?, ¿qué es lo que está bien? ¿por dónde empiezo? Frente a estos interrogantes es importante que el docente no brinde respuestas cerradas, que no elija por el alumno las alternativas más convenientes, sino por el contrario fomente un aprendizaje activo basado en una construcción conjunta entre alumno y profesor para aprender a pensar y a actuar juntos: copensar y cooperar. Promover la toma de conciencia del alumno en cuanto a las operaciones y decisiones mentales que realiza cuando resuelve una tarea lo que a futuro permitirá el desarrollo de su autonomía.

El profesor también formulará preguntas al alumno que permitan promover el pensamiento y guiar acciones. Esto favorece una práctica reflexiva y un andamiaje que puede retirarse a medida que el alumno progresa en la adquisición de cierta autonomía. La formulación de preguntas se consolida entonces como una herramienta muy valiosa que puede permitirle al alumno conectar sus conocimientos previos con los nuevos. La pregunta puede formularse:

- Para iniciar la clase. Se trata de dar sentido a lo nuevo que se está por aprender en relación con el trabajo realizado en clases anteriores. Contextualizar,

- Durante el desarrollo de la clase. Incitan la búsqueda de soluciones frente a un problema. Preguntas que impulsan búsquedas,

- Preguntas finales. Favorecen procesos de síntesis y conclusiones. Anticipan el tema de la clase siguiente

Las preguntas dirigidas no solo al campo de la cognición (conceptos, información) sino también incluyendo aquellas referidas a la metacognición para que los estudiantes reconozcan cómo han pensado, qué relaciones establecieron para alcanzar así mejores procesos de pensamiento.

\section{¿QUÉ SIGNIFICA ESTUDIAR EN CASA?}

La carga horaria excesiva se visualiza en el proyecto de gestión como una problemática actual que atraviesan los alumnos del BBA. Las jornadas extendidas dificultan realizar por parte de los alumnos un trabajo sostenido y regular fuera del colegio.

Muchas veces que el profesor indique "estudiá para la próxima clase" no representa una consigna muy alentadora para muchos alumnos, la palabra estudiar se vacía de contenido y encuentra al alumno en su casa sin saber qué hacer. Cabe destacar que estudiar puede significar para cada alumno una cosa diferente, para algunos será realizar una práctica repetitiva (acumular "pasadas"), para otros será avanzar con la lectura, para otros será tocar a último momento antes de la clase, subir la velocidad, etc. Es de suma importancia indicar opciones de "estudiar" personalizadas según las dificultades propias de cada alumno.

La reflexión, el estudio consciente parece disminuir sin la presencia del docente, el alumno suele expresar su incertidumbre frente a la partitura. Se propone entonces efectuar una bitácora de trabajo que le permita al alumno expresar de manera puntal y resumida con palabras escritas experiencias de estudio. Describir el trabajo realizado, organización, administración del tiempo, elecciones de recursos, dificultades, sensaciones positivas o negativas, curiosidad, creatividad, desgano, fragilidad frente a las dificultades, falta de metas, etc. 
El texto escrito permite que el alumno exprese ordenadamente las ideas, tome conciencia de ellas situando al lenguaje en su función de andamiaje del pensamiento. Narrar se constituye así como una forma de pensar y de crear sentidos.

A partir de esto, las tareas entre clase y clase no deben ser asignadas exclusivamente por el profesor, sino en forma compartida con un alumno que es capaz de reflexionar acerca de las dificultades que debe superar. La construcción en forma conjunta de la problemática a resolver permitirá no sólo el trabajo en conjunto para la búsqueda de posibles soluciones sino también seguir conociendo el problema.

El alumno podrá aprender a supervisar su propia práctica fuera de la clase, con la guía de un profesor que pueda brindar también posibles estrategias previendo dificultades que pudieran presentarse. Desde esta concepción el error es motor del aprendizaje puesto que conduce a nuevas reflexiones. Desde una concepción más tradicional lo único que se aprende de los errores es a evitarlos. Tradicionalmente las metodologías utilizadas en las clases de instrumento proponen como meta realizar algo "bien", reproducir con éxito un determinado producto sonoro y las estrategias para alcanzar estos fines las gestiona solamente el profesor. Desde una perspectiva constructivista la meta es reflexionar sobre "cómo se hace" o en todo caso por qué se hace "bien o mal", impulsar procesos de autorregulación que permitan saber al alumno cuándo comprende, qué comprende y qué no, qué necesita para comprender. La tarea docente consiste en buscar la activación cognitiva del alumno frente a lo que se va a hacer y que finalmente el alumno entienda cuál es la mejor solución y por qué.

La selección del material apuntará a un repertorio que abarque la mayor cantidad de preferencias musicales transitando por un amplio repertorio que trascienda la dicotomía popular-académico e incluya el "repertorio tradicional" de diferentes periodos históricos desde el barroco hasta nuestros días, y música argentina y latinoamericana. Incluya además de las formas más convencionales de uso instrumental, diversas modalidades de ejecutante como el ejecutante arreglador, integrante de agrupaciones musicales, el instrumentista acompañante, etc.

El material debe brindar al alumno la sensación de competencia. Si se plantea una actividad muy alejada de su zona de desarrollo próximo, fuera de su alcance es probable que no pueda realizarla satisfactoriamente lo que contribuirá a la formación de inseguridad y frustración. De la misma manera la minusvaloración del potencial de aprendizaje de los estudiantes conlleva al uso de un repertorio acotado y repetitivo generando aburrimiento y falta de implicancia. Esto demanda a los docentes realizar constantes ajustes, ensayar diferentes propuestas y de ser necesario saber desestimar y reemplazar el material de trabajo.

Lo que puede resultar familiar o cercano para un alumno puede ser el punto de partida para posteriormente ir ampliando las fronteras de lo conocido. Es de suma importancia entender que el espacio curricular de instrumento es un espacio formativo por lo tanto es responsabilidad del docente administrar la elección de obras musicales con cierto grado de secuenciación y proyección, en este sentido es quien tiene el criterio pedagógico necesario para determinar que obras son más convenientes trabajar según el recorrido particular del alumno y en vistas de los requerimientos instrumentales. El docente ofrece un paquete de obras variadas que comparten algún criterio de agrupación como puede ser textural, rítmico, estilístico, expresivo, de articulación, etc.; de esta selección previa el alumno a su vez elige según sus propias preferencias y gustos.

Los alumnos abordarán las obras incluyendo:

- Una reflexión previa. Análisis en conjunto de los elementos constitutivos de la obra. Encontrar lógicas internas, relaciones entre los elementos, previo a la puesta en acto.

- Una reflexión en la acción. Durante la ejecución/interpretación, para ir ratificando aquellos aspectos analizados de antemano generando un pensamiento anticipado y expectativas acerca de posibles modos de continuidad musical.

- Una reflexión sobre la acción. Describiendo la experiencia obtenida al haber finalizado la acción. 
El conocimiento musical se construye como una experiencia intersubjetiva. El intercambio permite visualizar otras formas de aprender, "cómo aprenden mis compañeros", "cómo aprenden mis profesores". Propiciar espacios de encuentro permite descubrir diferentes formas de proceder. Espacios abiertos que estimulan el encuentro con otros alumnos del mismo profesor, con otro/s profesor/es y sus alumnos, con otros lenguajes (artes visuales, audiovisual, teatro, danza) en contextos diversos como pueden ser una situación de clase, asistiendo a conciertos de profesores, muestras, proyecciones, audiciones de alumnos, conciertos didácticos, etc. "Explorar diferentes lenguajes artísticos en igualdad de condiciones para varones y mujeres, erradicando prejuicios habitualmente establecidos". (Ley 26.150 de ESI, 2006)

Compartir experiencias acerca del proceso de construcción de una obra, el acto performático, la búsqueda de significados y la forma de comunicarlos, se transforma en una práctica integradora y significativa.

\section{EVALUACión}

La evaluación educativa es una actividad sistemática continua y crítica del proceso enseñanza- aprendizaje. Es un aprendizaje en sí misma tanto para los alumnos como para los docentes. Este concepto de evaluación abre la posibilidad de recrear las normas existentes o de definir nuevas, y adquiere un sentido constructivo en oposición a definiciones de evaluación enmarcadas en un plano más normativo, donde la evaluación sólo se refiere a si las acciones realizadas se ajustan o no a un patrón normativo que representa un modelo ideal. Este sentido constructivo implica participar en la construcción de un tipo de conocimiento axiológico integrado a la actividad educativa con el objetivo de la mejora continua, incluyendo información sobre el proceso de aprendizaje y sobre los factores personales y ambientales que inciden. Implica entender a la evaluación como una práctica cotidiana en la experiencia áulica que realizan todos los actores, que afecta a la institución en su conjunto y que potenciará el desarrollo de todos sus miembros.

Es de suma importancia que a partir de la información que brinda la evaluación de tipo formativa entendida coma una actividad continua y sistemática que considera todo el proceso educativo, se impulse un proceso de retroalimentación para que tanto alumnos como docentes revisen, resignifiquen su trabajo y tomen decisiones pertinentes.

Para no generar impactos negativos como temor o inseguridad en el alumno, el docente debe aportar transparencia en cuanto a las expectativas de aprendizaje y los criterios de evaluación y definir los niveles de calidad de cada uno de los desempeños. Evaluar supone la existencia de un desafío comunicacional para generar alumnos motivados. Una retroalimentación positiva y constructiva por parte del docente, que le indique al alumno cómo progresar y que enfatice los logros promoverá la motivación por aprender.

Con el fin de que los estudiantes puedan promover su aprendizaje y forjar una conciencia metacognitiva, es importante estimular la autoevaluación en los alumnos, fomentando una motivación intrínseca en la que el alumno valore positivamente sus logros y no sólo sus dificultades. La evaluación no es exclusiva del docente sino que pueden participar los propios educandos en forma colaborativa, la coevaluación entre pares puede ser una herramienta útil en las instancias de aula abierta. El docente busca establecer vínculos positivos, solidarios y de respeto entre los alumnos, y propicia la creación de un clima de espontaneidad y confianza. Al desarrollar la capacidad de observación al analizar los trabajos de los compañeros también se le permite al alumno aprender a analizar su propia tarea.

$\mathrm{Al}$ desarrollar las capacidades de autoevaluación y coevaluación se convierten en sujetos más reflexivos, autónomos y hábiles para gestionar su aprendizaje, y resolver las dificultades que puedan presentarse.

El trimestral representa una instancia de evaluación más formalizada de tipo sumativa. De acuerdo con los lineamientos departamentales se estipula que el alumno rinda dos obras pianísticas flexibilizando la propuesta según la longitud o dificultad de la misma. Es importante que los estudiantes lleguen a esta instancia con un planteointerpretativo, es decir una propuesta, un esbozo de interpretación, que sin ser el definitivo incluirá: 
- la ejecución de la totalidad evitando tocar fragmentos desarticulados,

- la continuidad discursiva en la puesta en acto

- compromiso en la utilización de competencias expresivas y comunicativas.

Para ser coherentes con el eje central de esta propuesta es importante que en la evaluación trimestral además de ejecutar la obra respetando los criterios mencionados, el alumno realice comentarios, narre brevemente cómo fue el proceso de construcción del planteo interpretativo, con qué dificultades se encontró, qué opciones de resolución puso en juego, qué cosas faltan desarrollar, qué trabajo técnico emergió de las necesidades expresivas y musicales, qué aprendizajes movilizó la experiencia de tocar frente a sus pares y otros docentes, etc.

Para concluir, es importante vislumbrar los alcances de una clase de instrumento. Cómo partiendo de problemáticas propias de la interpretación musical y del campo específico, se puede colaborar en la formación de ciudadanos más reflexivos, comunicativos y sensibles.

\section{ReFERENCIAS}

Aguerre, A. (2017). BBA Texturas Reunidas. Proyecto académico y de gestión 18-22. La Plata: Bachillerato de BA. UNLP Aguerre, A. (2007). La decodificación e interpretación de textos musicales en la enseñanza instrumental. Ideas para una nueva educación II. La Plata: Bachillerato de BA. UNLP.

Alvarez, A. y Del Rio Pereda, P. (1990). Educación y desarrollo. La teoría de Vygotsky y la zona de desarrollo próximo. En J. Palacios González, A. Marchesi Ullastres y C. Coll (comp.), Desarrollo psicológico y educación (vol. 2, p. 93-119). Recuperado de https://mcesuvaq.files.wordpress.com/2013/08/9-educacic3b3n-y-desarrollo.pdf

Arturi, M. y Acevedo, A. M. (compiladores) (2004). Bachillerato de Bellas Artes. Ideas para una nueva educación. La Plata: Edición del Bachillerato de Bellas Artes de la U.N.L.P

Arturi, M. y Acevedo, A. M. (compiladores) (2007). Bachillerato de Bellas Artes. Ideas para una nueva educación II. La Plata: Edición del Bachillerato de Bellas Artes de la U.N.L.P

Bertone, F. (2016). Proyecto de gestión Departamental. Departamento de discursos musicales. La Plata: Universidad Nacional de La Plata.

Competencia para aprender a aprender. Marco teórico (2012) Recuperado de http://ediagnostikoak.net/edweb/cas/m ateriales-informativos/ED_marko_teorikoak/Aprender_a_aprender.pdf

Domingo Roge, A. (s.f.) El profesional reflexivo (D.A. Schön) Descripción de las tres fases del pensamiento práctico. Recuperado de http://www.academia.edu/28190466/El_profesional_reflexivo

Efrón, R. (1996). Subjetividad y adolescencia. En I. Konterllnik y C. Jacinto (comp.), Adolescencia, pobreza, educación $y$ trabajo. Buenos Aires: Losada - UNICEF.

Feldman, D. (2015). Para definir el contenido notas y variaciones sobre el tema en la universidad. Recuperado de htt $\mathrm{p}: / /$ revistas.unlp.edu.ar/TrayectoriasUniversitarias

Feldman, D. y Palamidessi, M. (2001). Programación de la enseñanza en la universidad. Problemas y enfoques. Colección Universidad y Educación. Serie Formación Docente Nº1. Buenos Aires: Editorial de la Universidad Nacional de General Sarmiento.

Hemsy de Gainza, V. y Kesselman, S. (2003). Música y Eutonía. El cuerpo en estado de arte. Buenos Aires: Editorial Lumen.

Ministerio de Educación (2008). Lineamientos Curriculares para la Educación Sexual Integral. Programa Nacional de Educación Sexual Integral. Ley Nacional N ${ }^{\circ}$ 26.150. Buenos Aires: Ministerio de Educación.

Lanz, Z. M. (2006). Aprendizaje autorregulado: el lugar de la cognición, la metacognición y la motivación. Estudios Pedagógicos, XXXII(2), 121-132. Recuperado de http://www.redalyc.org/articulo.oa?id=173514131006

Ley 26.150: Programa Nacional de Educación Sexual Integral Ley 26.150. Boletín Oficial de la República Argentina, Buenos Aires, Argentina, 24 de octubre de 2006. 
Lluch Molins, L. y Portillo Vidiella, M. C. (2018). La competencia de aprender a aprender en el marco de la educación superior. Revista Iberoamericana De Educación, 78(2), 59-76. Recuperado de https://rieoei.org/RIE/article/vi ew/3183

Monereo, C., Pozo, J. I. y Castelló, M. (2001). La enseñanza de estrategias de aprendizaje en el contexto escolar. En C. Coll, J. Palacios y A. Marchesi (Coord.). Psicología de la educación escolar. Madrid: Editorial Alianza. Recuperado de https://www.researchgate.net/publication/261082782_La_ensenanza_de_estrategias_de_apre ndizaje_en_el_contexto_escolar

Moral Santaella, C. (2008). Aprender a pensar- Aprender a aprender. Habilidades de pensamiento y aprendizaje autorregulado. Bordón. Revista de Pedagogía, 60(2), 123-137. Recuperado de https://recyt.fecyt.es/index.php/ BORDON/article/view/29019

Morchio, I. L. (Coord.) (2015). Aprender a aprender como meta de la Educación Superior. Desde la comprensión de cómo aprende el alumno universitario a la promoción del aprendizaje autorregulado. Buenos Aires, Argentina: Editorial Teseo. Recuperado de http://bdigital.uncu.edu.ar/8360

Pozo, J. I. y Monereo, C. (2010). Aprender a aprender cuando los contenidos son el medio. Aula de innovación educativa, 190, 35-37. Recuperado de https://repositorio.uam.es/bitstream/handle/10486/668584/aprender_ pozo_aie_2010.pdf?sequence $=1$

Ramírez, S. (2013). Proyecto académico y de gestión Periodo 2014- 2018. La Plata: Bachillerato de Bellas Artes Prof. Francisco A. De Santo. Universidad Nacional de La Plata

Reyes Carretero Torres, M. y Fuentes Agustí, M. (2010). La competencia de aprender a aprender. Aula de innovación educativa, 192, 7-10. Recuperado de https://core.ac.uk/download/pdf/132092907.pdf

Stubley, E. V. (1992). Fundamentos filosóficos. En R. Colwell (coord.), Handbook of Research on Music Teaching and Learning: a project of the Music Educators National Conference. España: Editorial Schirmer Books.

Torrado, J. A. y Braga, M. (2016). Gestionando la comunicación emocional desde los sonidos: una oportunidad para la música en la educación del siglo XXI. Pirineos.Revista de la Consejería de Educación de la Embajada de España en Andorra, 12, 112-115. Recuperado de https://dialnet.unirioja.es/servlet $/$ ejemplar $?$ codigo $=450320 \&$ info $=0$ pen_link_ejemplar

Torrado, J. A. y Pozo, J. I. (2008). Metas y estrategias para una práctica constructiva en la enseñanza instrumental. Culture and Education, 20(1), 35-48. Recuperado de http://hdl.handle.net/10486/665297

Torrado, J. A., Casas, A. y Pozo, J. I. (2005). Las culturas de la educación musical: aprendiendo a interpretar un instrumento. Estudios de psicologia, 6(2), 259-269. Recuperado de http://hdl.handle.net/10486/666792

Torrado, J. A., Casas, A. y Pozo, J. I. (2008). El aprendizaje y la enseñanza de la interpretación musical: cambiando las concepciones y las prácticas. Culture and Education, 20(1), 5-15. Recuperado de : http://hdl.handle.net/10 $486 / 665296$

\section{BY-NC-SA}

